\title{
O NÍVEL DE COLESTEROL INFLUENCIA A QUANTIDADE DE FOLÍCULOS NA PUNÇÃO FOLICULAR DE VACAS DE CORTE
}

\section{CHOLESTEROL LEVELS AFFECT THE QUANTITY OF FOLLICLES IN BEEF CATTLE OVUM PICK-UP}

\author{
Pfeifer, L.F.M. ${ }^{1 *}$, I. Pivato ${ }^{3}$, R. Rumpf ${ }^{4}$, N.J.L. Dionello¹, A. Schneider², M.A. Goulart ${ }^{2}$ \\ e M.N. Corrêa ${ }^{2}$
}

\begin{abstract}
${ }^{1}$ Departamento de Zootecnia. Faculdade de Agronomia. Universidade Federal de Pelotas (UFPel). Campus Universitário s/n. Caixa Postal 354. CEP 96010-900. Pelotas/RS. Brasil. *Correspondencia: Ifmpfeifer@terra.com.br

${ }^{2}$ Departamento de Clínicas Veterinária. Faculdade de Veterinária. Universidade Federal de Pelotas (UFPel). Campus Universitário s/n. Caixa Postal 354. CEP 96010-900. Pelotas/RS. Brasil.

${ }^{3}$ Companhia Integrada de Desenvolvimento Agricola de Santa Catarina. Rua Marechal Deodoro da Fonseca $n^{\circ}$ 4500. Bairro Warnow. Caixa Postal 87. CEP 89130-000. Indaial/SC. Brasil.

${ }^{4}$ EMBRAPA. Recursos Genéticos e Biotecnologia. Parque Estação Biológica. Av. W5 Norte (final). Caixa Postal 02372. CEP 70770-900. Brasília/DF. Brasil.
\end{abstract}

\section{PalaVRas chaVe ADICIONAIS \\ Qualidade de ovócitos. Foliculogênese.}

\section{RESUMO}

O objetivo deste trabalho foi avaliar o número de folículos por punção folicular (PF), a quantidade e a qualidade de ovócitos oriundos de vacas com diferentes níveis séricos de colesterol. Para a realização deste experimento foram utilizadas 5 vacas mestiças. Antes do início do experimento, todas as vacas receberam por 8 dias um dispositivo intravaginal liberador de progesterona e dois dias antes da retirada dos dispositivos todas as vacas receberam $0,150 \mathrm{mg}$ de d-cloprostenol. As sessões de PF ocorreram a cada 4 dias, totalizando 6 sessões. Os animais receberam $0,150 \mathrm{mg}$ de d-cloprostenol a cada 8 dias, juntamente com a troca dos dispositivos. Após avaliação do nível sérico de colesterol, as vacas foram divididas em dois grupos: G1 (colesterol<50 mg/dl) e G2 (colesterol $>50 \mathrm{mg} / \mathrm{dl})$. Foi registrado uma diferença $(p<0,01)$ entre o $G 1$ e o $G 2$ quanto ao número de folículos/punção, 9,66 e 12,6, respectivamente, não sendo observado diferença $(p>0,05)$ no número de ovócitos coletados e na qualidade dos mesmos. Verificou-se que vacas com níveis de colesterol superior à $50 \mathrm{mg} / \mathrm{dl}$ apresentaram um maior número de folículos aptos à punção.

\section{AdDitionAL KEYWORDS \\ Oocyte quality. Folliculogenesis.}

\section{SUMMARY}

The aim of this work was to evaluate the number of follicles able to ovum pick up (OPU), the quantity, and quality of oocytes from donors showing different blood levels of cholesterol. For this experiment 5 crossbreed cows were used. Before the beginning of the experiment all cows received an intravaginal progesterone releasing device for 8 days. Two days before the device withdraw all cows received $0.150 \mathrm{mg}$ of $\mathrm{d}$ cloprostenol. The cows were submitted to ovum pick up each 4 days in a total of 6 sections. The cows were injected with $0.150 \mathrm{mg}$ of $\mathrm{d}$ cloprostenol each 8 days, at the moment of the device change. After the evaluation of blood cholesterol, cows were divided in two groups: G1 (cholesterol $<50 \mathrm{mg} / \mathrm{dl}$ ) and $\mathrm{G} 2$ (cholesterol $>50$ $\mathrm{mg} / \mathrm{dl})$. There was a difference $(\mathrm{p}<0.01)$ between G1 and G2 in the number of follicles/punction, 9.66 and 12.6, respectively, but we didn't observe difference in the number of recovered oocytes and its quality. These results have shown that cows with blood levels of cholesterol higher than $50 \mathrm{mg} / \mathrm{dl}$ have a higher number of follicles able to punction.

Arch. Zootec. 58 (221): 153-156. 2009. 


\section{INTRODUÇÃO}

O controle do crescimento folicular em ruminantes depende de uma série de fatores que atuam tanto em nível ovariano quanto hipofisário (Lee et al., 2001). Dentre estes fatores, estão os relacionados a nutrição, que podem influenciar o desenvolvimento folicular e a qualidade ovocitária (Webb et al., 2004). Há evidências de que pequenas mudanças no plano alimentar ou variações individuais no consumo podem afetar a dinâmica folicular, mesmo sem alterar a concentração sérica de gonadotrofinas hipofisiárias (Holmes et al., 2002).

Neste âmbito, o estudo da interação de alguns marcadores metabólicos com hormônios da reprodução e função ovariana se torna interessante. Um bom candidato é o colesterol, que é precursor dos hormônios esteróides (Grummer e Carroll, 1991) e está indiretamente relacionado com a regulação do eixo hipotálamico-hipofisiário e da foliculogênese, podendo afetar também a fertilidade dos animais.

Este estudo teve por objetivo avaliar o número de folículos aptos a punção e a qualidade de ovócitos de vacas de corte com diferentes níveis de colesterol sérico.

\section{MATERIAISEMÉTODOS}

Para a realização deste experimento foram utilizadas 5 vacas mestiças, com condição corporal 3 , em regime de pastagem recebendo suplementação mineral. Antes do início do experimento todos animais foram submetidos ao exame ginecológico por ultrassonografia para confirmação de ciclicidade.

Após todas as vacas receberam um dispositivo intravaginal liberador de progesterona (CIDR $\AA$, 1,9 $\mathrm{g}$ de progesterona) por 8 dias e 2 dias antes da retirada dos dispositivos receberam $0,150 \mathrm{mg}$ de d-cloprostenol (Prostaglandina Tortuga ${ }^{\circledR}$, im). No dia da retirada dos dispositivos os animais foram submetidos à punção folicular (PF), sendo realizadas mais 5 sessões a cada 4 dias durante o experimento. Após a primeira PF, as vacas receberam um dispositivo intravaginal reutilizado, que foi substituído a cada 8 dias, junto com a aplicação de dcloprostenol, com o objetivo de mimetizar a fase luteal precoce do ciclo estral.

O método de PF utilizado foi o descrito por Petyim et al. (2003). As PFs foram realizadas via transvaginal com um aparelho de ultrasom equipado com sonda setorial convexa transvaginal de $5 \mathrm{MHz}$, adaptado a um sistema de agulhas descartáveis e um sistema de vácuo. Foram puncionados todos os folículos com diâmetro acima de $3 \mathrm{~mm}$.

Os ovócitos aspirados foram avaliados em lupa estereomicroscópica, sendo os classificados nas categorias I e II considerados como de boa qualidade e nas categorias III e IV como de má qualidade, segundo Leibfried e First (1979).

Foram coletadas amostras de sangue para análise de progesterona em todas as sessões de PF, sendo a concentração determinada pela técnica de radioimunoensaio em fase sólida com I ${ }^{123}$ (Coat-A-Count ${ }^{\mathbb{R}}$, Los Angeles, CA, USA).

A avaliação dos níveis de colesterol foi feita utilizando amostras coletadas no momento da $1^{\circ} \mathrm{PF}$ e analisadas através de reações colorimétricas $\left(\mathrm{LABTEST}^{\circledR}\right)$ quantificadas em espectrofotômetro.

Após a avaliação do colesterol os animais foram divididos em 2 grupos: grupo 1 (G1, $\mathrm{n}=3$ ) animais que apresentaram colesterol dentro do padrão fisiológico $(<50 \mathrm{mg} / \mathrm{dl})$ e grupo $2(\mathrm{G} 2, \mathrm{n}=2)$ acima do padrão fisiológico (>50 mg/dl) (Wittwer, 2000).

As análises estatísticas foram realizadas utilizando os modelos de análise de variância do programa SAS ${ }^{\circledR}$ (1991).

\section{RESULTADOSEDISCUSSÃO}

Foram aspirados 271 folículos e recuperados 164 ovócitos durante todo o período experimental, com uma taxa de recuperação ovocitária de $60,5 \%$. O G2 apresentou maior $(\mathrm{p}<0,01)$ número folículos/punção/vaca 
$(12,6 \pm 0,64)$ do que o G1 $(9,66 \pm 0,57)$. Apesar de ter sido registrada diferença na quantidade de folículos, o mesmo não foi registrado $(\mathrm{p}>0,05)$ com a quantidade de ovócitos coletados/punção/vaca, sendo de 5,9 \pm 1 e de 7,5 $\pm 1,25$ ovócitos para o $\mathrm{G} 1$ e G2, respectivamente.

Apesar de alguns autores atribuirem diferenças no perfil metabólico e na dieta como fatores que podem influenciar a qualidade dos gametas femininos (Boland et al., 2001), neste estudo não foi registrado diferença $(\mathrm{p}>0,05)$ na qualidade ovocitária entre os grupos. Foi observado uma média de $3,42 \pm 0,54$ e $2,67 \pm 0,6$ ovócitos de qualidade I e II/vaca/punção e 3,43 $\pm 0,49$ e $5,1 \pm 1$ ovócitos de qualidade III e IV/punção/ vaca para oG1 eG2, respectivamente $(\mathrm{p}>0,05)$.

Visto que o colesterol é o precursor para síntese de hormônios esteróides (Grummer e Carroll, 1991), os animais que apresentaram maiores níveis de colesterol podem ter apresentado maior esteroidogênese em nível folicular (Kronfeld et al., 1980). Segundo

\section{BIBLIOGRAFIA}

Boland, M.P., P. Lonergan and D. O'Callaghan. 2001. Effect of nutrition on endocrine parameters, ovarian physiology and oocyte and embryo development. Theriogenology, 55: 1323-1340.

Grummer, R.R. e D.J. Carroll. 1991. Effects of dietary fat on metabolic disorders and reproductive performance of dairy cattle. $J$. Anim. Sci., 69: 3838-3852.

Holmes, C.W., I.M. Brookes, D.J. Garrick, D.D.S. Mackenzie, T.J. Parkinson and G.F. Wilson. 2002. Milk production from pasture. Ed. Butterworth-Heinemann. Palmerston North. New Zealand.

Kastelic, J.P. 2004. Folliculogenesis in cattle. In: $1^{\circ}$ Simpósio Internacional de Reprodução Animal Aplicada, Anais ..., Londrina, PR. p. 17-25.

Kronfeld, D.S., S. Donoghue, J.M. Naylor, K. Johnson and C.A. Bradley. 1980. Metabolic effects of feeding protected tallow to dairy cows. J. Dairy Sci., 63: 545-552.

Lee, W.S., F. Otsuka, R.K. Moore and S. Shimasaki.
Leroy et al. (2003) há uma alta correlação entre o nível sérico e intrafolicular de colesterol, e desta forma, o nível de inibina e estradiol podem estar elevados nos animais do G2. Este aumento do nível de hormônios esteróides pode estar aumentando o bloqueio do eixo hipotalâmico-hipofisário (Kastelic, 2004), reduzindo a ação dos mecanismos para seleção do folículo dominante e conseqüente atresia folicular.

O nível plasmático médio de progesterona registrado neste experimento foi de 1,58 $\mathrm{ng} / \mathrm{ml}$ durante as 5 sessões de punção, não havendo diferença entre os animais $(\mathrm{p}>0,05)$. Estes níveis são compatíveis com os registrados por Sartori et al. (2004) no período luteal recente.

\section{CONCLUSÃO}

A partir dos resultados verificamos que vacas com níveis de colesterol superior a 50 $\mathrm{mg} / \mathrm{dl}$ apresentaram um maior número de folículos aptos à punção folicular.

2001. Effect of bone morphogenetic protein-7 on folliculogenesis and ovulation in the rat. Biol. Reprod., 65: 994-999.

Leibfried-Rutledge, M.L. and N.L. First. 1979. Characterization of bovine follicular oocytes and their ability to mature in vitro. J. Anim. Sci., 48: $76-86$.

Leroy, J.L.M.R., T. Vanholder, A. Van Soom, G. Opsomer, P. Bols and A. de Kruif. 2003. Effect of oleic acid during in vitro maturation on fertilisation, first cleavage and embryo development of bovine cumulus-oocyte-complexes. Reprod. Domest. Anim., 38: 328 (abstr.).

Petyim, S., R. Bage, T. Hallap, A.S. Bergqvist, H. Rodriguez-Martínez and B. Larsson. 2003. Two different schemes of twice-weekly ovum pickup in dairy heifers: effects on oocyte recovery and ovarian function. Theriogenology, 60: 175188.

Sartori, R., J.M. Haughian, R.D. Shaver, G.J.M. Rosa and M.C. Wiltbank. 2004. Comparison of ovarian function and circulating steroids in 
PFEIFER, PIVATO, RUMPF, DIONELLO, SCHNEIDER, GOULART E CORREAA

estrous cycles of holstein heifers and lactating cows. J. Dairy Sci., 87: 905-920.

SAS ${ }^{\circledR}$. 1991. SAS/STAT User's Guide (Release 6.03). SAS Inst. Inc. Cary, NC.

Webb, R., P.C. Garnsworthy, J.G. Gong and D.G. Armstrong. 2004. Control of follicular growth: Local interactions and nutritional influences. J. Anim. Sci., 82: E63-E74.
Wittwer, F. 2000. Marcadores bioquímicos no controle de problemas metabólico nutricionais em gado de leite. Em: Gonzalez, F.H.D., J. Barcelos, H.O. Patino e L.A. Ribeiro (Eds.). Perfil metabólico em ruminantes: seu uso em nutrição e doenças nutricionais. Gráfica da Universidade Federal do Rio Grande do Sul. Porto Alegre, Brasil. 